\title{
ON A NEW APPROACH TO THE ANALYSIS OF STATIONARY INVENTORY PROBLEMS*
}

\author{
Oscar A. Z. Leneman \\ Massachusetts Institute of Technology \\ Lincoln Laboratoryt \\ Lexington. Wassachuset $t$ \\ and \\ Frederick J. Beutler \\ Computer, Information a Control Engineering Program \\ The University of Uichigan \\ Ann Arbor, Michigan
}

\begin{abstract}
ABST RACT
The intent of this paper is to demonstrate that the theory of stationary point processes is a useful tool for the analysis of stationary inventory systems. In conventional inventory theory, the equilibrium distributions for a specified inventory policy are obtained, whenever possible, by recursive or limiting procedures, or both. A different and more direct approach, based on stationary point processes, is proposed here. The time instants at which stock delivery is effected are viewed as points of the stationary point process, which possesses uniform statistical properties on the entire real axis; hence the equilibrium statistics of the inventory process can be calculated directly. In order to best illustrate this approach, various examples are given, including some that constitute new results.
\end{abstract}

\section{INTRODUCTION}

Once a class of inventory policies is specified, the study of the inventory model becomes a study of the associated stochastic process. Quite often one seeks to choose within this class the policy which minimizes the average long run costs and as a result one is interested in studying the stationary behavior of the inventory model. This type of study is, in general, difficult and the usual approach, utilizing recursive and limiting procedures $[1,4,5]$ is often limited.

The intent of this paper is to show by examples and illustrations that such stationary state inventory studies are systematically facilitated by the use of some recent results from the theory of stationary point processes (hereafter abbreviated s.p.p.). An s.p.p. possesses uniform stationarity properties over the entire real axis, so that an inventory process based

\footnotetext{
*This study was sponsored by Lincoln Laboratory, a center for research operated by the Massachusetts Institute of Technology with support from the U.S. Air Force and from the U.S. Advanced Research Projects Agency. Support also furnished by the National Aeronautics and Space Administration under Research Grant NsG-2 -59. This article was originally drafted by $O$. A. Z. Leneman and revised for publication by F. J. Beutler during the period of leave in India of the first author.

tOperated with support from the U.S. Advanced Research Projects Agency.
} 
on an s.p.p. will be in the same statistical state for all times $t,-\infty<t<+\infty$. The statistics of the inventory process can thus be calculated directly; the final result does not involve $t$, and no asymptotic techniques are required.

For the reader unfamiliar with s.p.p. we state some of the s.p.p. definitions and properties, mentioning in particular those needed for the examples that follow. A detailed exposition of s.p.p. is found elsewhere $[2,3]$, and the reader desiring proofs or further information should refer to $[2,3]$. A random point process $\left\{t_{n}\right\}$ is a denumerable ordered family of random variables that may be regarded as points (occurrences) on the time axis. In particular, the points $t_{n}$ can be related to inventory processes by taking these points to be the instants at which orders are placed for each successive inventory cycle.

An s.p.p. is a random point process meeting certain statistical uniformity requirements. For any finite set of intervals on the real axis, the joint probability distribution function of numbers of points on these intervals must be invariant under any translation of the set of intervals. This definition of stationarity is equivalent to any one of a number of other possible definitions. For instance, if $\mathrm{L}_{k}(t)$ is the time interval extending from time $t$ to the $k^{\prime}$ th point on the right of $t$, stationarity of the point process is equivalent to the following: for any $n$, the joint distribution of the set of random variables $\left\{L_{1}(t), L_{2}(t), \ldots, L_{n}(t)\right\}$ does not depend on $t$. It should be noted that the intervals $x_{n}=t_{n+1}-t_{n}$ of an s.p.p. $\left\{t_{n}\right\}$ need be neither independent nor identically distributed.

The second definition of an s.p.p. makes it possible to define a probability distribution function $G_{n}(x)=P\left[L_{n}(t) \leq x\right]$. The random variable $L_{-n}(t)$, defined as the time interval extending from the $k^{\prime}$ th point to the left of $t$ to time $t$, has the same distribution function $G_{n}$ as $L_{n}$. It can be shown that the $G_{n}$ are absolutely continuous, and that the derivates $g_{n}$ possess limits from the right. The $G_{n}$ have a useful characterization for small argument. Suppose that $\lim _{x \rightarrow 0}\left[G_{2}(x) / G_{1}(x)\right]=0$, that is, the probability of two points (or more) in a small interval is negligible when compared with the probability of only one. Such an assumption is certainly appropriate to inventory processes, where reordering frequency is subject to natural limitations. We then have $\sum_{n=2}^{\infty} G_{n}(x)=o(x)$ and

$$
\mathrm{G}_{1}(\mathrm{x})=\beta \mathrm{x}+\alpha(\mathrm{x})
$$

in which $\beta$ represents the mean number of points per unit time. Because the sum of the $G_{n}$, $n \geq 2$, is negligible by comparison, $G_{1}(x)=P$ \{one point falls in $(t, t+x]$ for small $x$.

Another distribution function that will often appear in the examples below is $F_{k}(x)=$ $P\left[L_{k}(t) \leq x \mid t=t_{n}\right.$ for some $\left.n\right]$. When the intervals $x_{n}$ are identically (but not necessarily independently) distributed, $F_{k}$ is the probability distribution function for $k$ successive intervals, that is

$$
F_{k}(x)=P\left[X_{1}+X_{2}+\cdots+X_{k} \leq x\right]
$$

We note further that $F_{k}$ obeys the relation

$$
F_{k}(x)=1-\beta^{-1} \sum_{j=1}^{k} g_{j}(x) \quad \text { for } x \geq 0
$$


and that we can use $F_{1}$ to show that the expectation

$$
E\left(X_{n}\right)=\beta^{-1}
$$

In the examples that follow, we shall often assume that the stock depletion rate $\lambda$, the time delay $\tau$ between ordering and delivery, and the amount $M$ delivered are independent random variables. Each of these correspond to realistic situations; for instance, the demand rate $\lambda$ is not precisely known in advance, and the delivered quantity $M$ may represent the imprecisely realized output of an entire plant or farm, and may be subject to an unpredictable number of rejected units on receiving inspection or a random number of units damaged in shipment. In any case, considering $\lambda, \tau$, and $\mathbf{M}$ to be random generalizes any analysis in which they are taken to be deterministic.

\section{EXAMPLE I: A CONTINUOUS TIME INVENTORY POLICY}

The following example will serve as an introduction. An amount of stock $M$ is reordered with instantaneous delivery whenever the inventory on hand reaches zero. The stock on hand after delivery is depleted at a linear rate $\lambda$ per unit time. The quantities $M$ and $\lambda$ are independent random variables selected from probability density functions $f_{M}$ and $f_{\lambda}$ for each inventory cycle. Assuming that the system has reached steady state, what is the probability density function of $X(t)$, the stock on hand at any given time $t$ ?

\section{SOLUTION}

The stock on hand is a stationary random process $X(t)$ as depicted in Figure 1. The instants of stock arrival at the random times $t_{n}$ constitute a stationary point process. Successive $t_{n}$ are separated by identically distributed intervals; if $T$ is such an interval

$$
\mathbf{T}=\frac{\mathbf{M}}{\lambda}
$$

The average number of stock arrivals per unit time is computed from (3) to be

$$
\beta=\frac{1}{E(T)}=\frac{1}{E(M) E(1 / \lambda)},
$$

where $E()$ denotes the expectation of the quantity in parentheses.

Let us introduce the notation $A_{0}=\{$ no stock arrival in $(t, t+y]\}$ and $A_{1}=$ \{one stock arrival in $(t+y, t+y+d y]\}$; these two events will be mentioned several times in what follows. Now

(6) $P\left[\{x<X(t) \leq x+d x\} \cap\left\{y<L_{1}(t) \leq y+d y\right\}\right]=P\left[\{x<X(t) \leq x+d x\} \cap A_{0} \cap A_{1}\right]$

$$
=\mathbf{P}\left(\mathbf{A}_{1}\right) \mathbf{P}\left[\{\mathbf{x}<\mathbf{X}(\mathbf{t}) \leq \mathbf{x}+d \mathbf{d x}\} \cap \mathbf{A}_{\mathbf{0}} \mid \mathbf{A}_{1}\right] \text {. }
$$

The first probability on the right is obtained from (1). If $A_{1}$ and $A_{0}$ are true, $X(t)$ must be given by $\lambda y$, and $T>y$ from the argument on interval distributions preceding (2). Hence the probability (6) becomes successively 


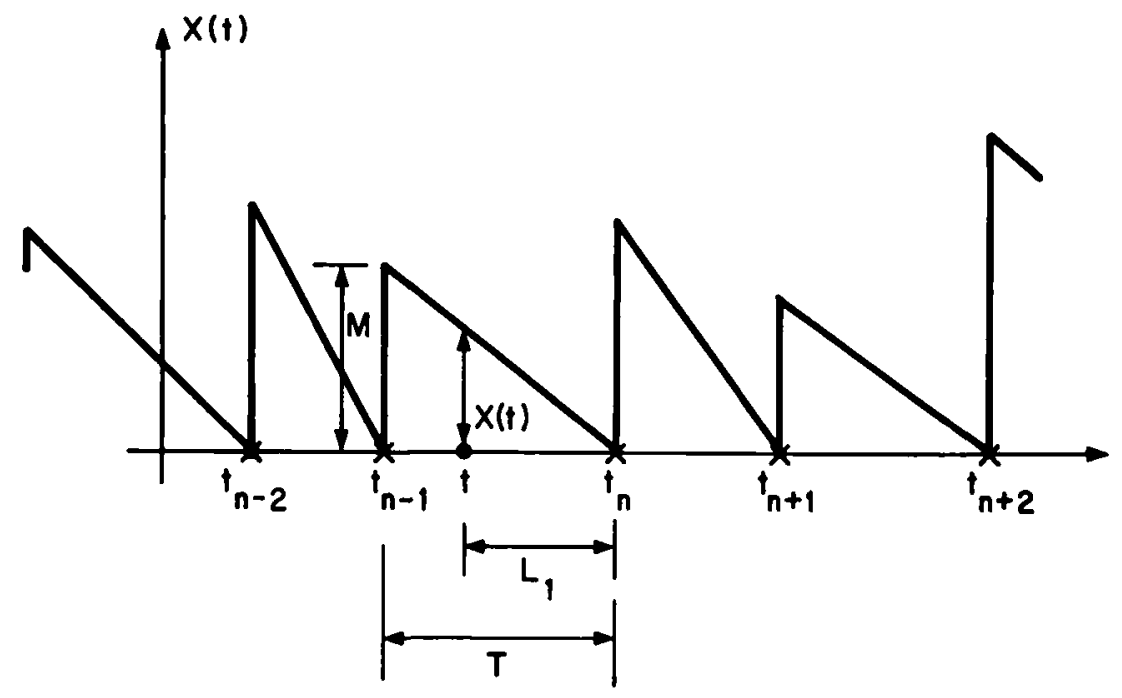

Figure 1. Inventory process with random linear depletion and instantaneous random reordering

(7) $\quad \beta$ dy $P[\{x<\lambda y \leq x+d x\} \cap\{T>y\}]=\beta$ dy $P\left[\left\{\frac{\mathbf{x}}{\mathbf{y}}<\lambda \leq \frac{\mathbf{x}}{\mathbf{y}}+\frac{\mathrm{dx}}{\mathbf{y}}\right\} \cap\left\{\frac{\mathbf{M}}{\lambda}>\mathbf{y}\right\}\right]$

$$
\begin{aligned}
& =\beta \text { dy } P\left[\frac{x}{y}<\lambda \leq \frac{x}{y}+\frac{d x}{y}\right] P[M>x] \\
& =\beta \operatorname{dy~} f_{\lambda}\left(\frac{x}{y}\right) \frac{d x}{y}\left[1-F_{M}(x)\right]
\end{aligned}
$$

in which $F_{M}$ is the probability distribution function for $M$. In order to find the density of $X(t)$, one integrates [see the left side of (6)] on y for the marginal density. Thus

$$
\begin{aligned}
& \mathbf{P}[\mathbf{x}<\mathbf{X}(\mathbf{t}) \leq \mathbf{x}+d \mathbf{x}]=\beta d \mathbf{x}\left[1-\mathbf{F}_{\mathbf{M}}(\mathrm{x})\right] \int_{0}^{\infty} \mathrm{y}^{-1} \mathbf{f}_{\lambda}\left(\frac{\mathrm{x}}{\mathrm{y}}\right) \mathrm{dy} \\
& =\beta \mathrm{dx}\left[1-\mathbf{F}_{\mathbf{M}}(\mathbf{x})\right] \int_{0}^{\infty} \mathbf{u}^{-1} \mathbf{f}_{\lambda}(\mathbf{u}) d \mathbf{u} \\
& =\beta d x\left[1-F_{M}(x)\right] E\left(\lambda^{-1}\right)
\end{aligned}
$$

which yields the rather simple final result

$$
f_{X}(x)=\left[1-F_{M}(x)\right] / E(M)
$$


The density of $\mathrm{X}(\mathrm{t})$ does not depend on the statistics of $\lambda$, because $\lambda$ affects the rate of ordering and variations in that rate, but not the amplitude ordered or the fraction of that amplitude on hand at a given instant.

\section{EXAMPLE II: A DISCRETE TIME (s,S) POLICY}

This example, which is well known in the literature [1], will also serve as an illustration of our approach. Note that it is no longer necessary to show the existence of the steady state (see [1], pp. 234-237) when using s.p.p. techniques.

Whenever the stock level falls below $s$, ordering is immediately enacted to raise the level to $S$ with immediate delivery; when the quantity in supply exceeds $s$, no ordering is done. A negative stock level is admissible, and can be viewed as an amount owed to consumption. Demands on the stock occur in discrete periods of duration $T_{0}$, the random demand sequence consisting of mutually independent identically distributed variates. The appearance of the stock level $X(t)$ as a function of time $t$ is then as shown in Figure 2. Our aim is again to determine the probability density $f_{X}$ of the stock on hand $X(t)$.

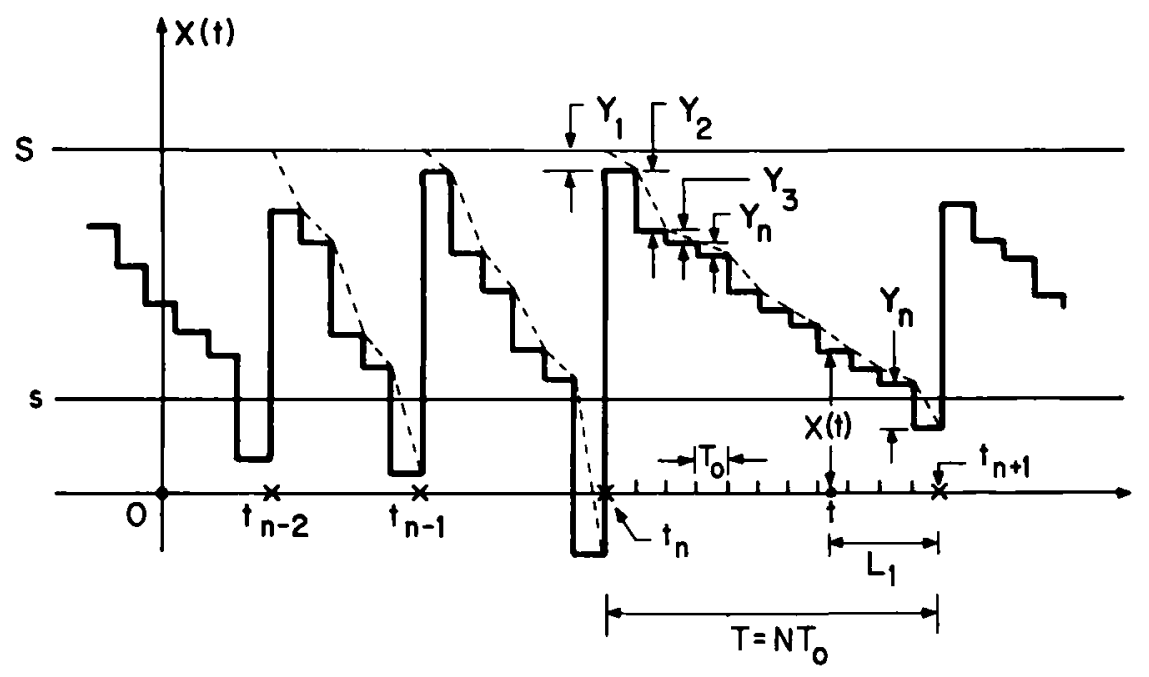

Figure 2. Inventory process with an $(s, S)$ policy

\section{SOLUTION}

The $t_{n}$ representing ordering times again constitute an s.p.p. with independent identically distributed intervals. Any time $t$ will be in some such interval, and we take the length of that interval to be the random variable

$$
\mathbf{T}=\mathrm{NT}_{\mathbf{O}}
$$

here $\mathrm{N}$ is the number of periods of length $\mathrm{T}_{0}$ required to reduce the stock from $\mathrm{S}$ (at the ordering time immediately preceding $t$ ) to $s$ or less. Let $Y_{1}, Y_{2}, \ldots$, be the random demands for this ordering interval. The total demand for the first $\mathrm{n}$ intervals is then defined to be $S_{n}=Y_{1}+Y_{2}+\cdots+Y_{n}$. This means that the random integer $N$ satisfies the inequalities

$$
\mathrm{S}_{\mathrm{N}-1}<\mathrm{S}-\mathrm{s} \leq \mathrm{S}_{\mathrm{N}}
$$


As in the preceding example, we shall need the average number of stock arrivals per unit time $\beta$. From the relationship (3) between $\beta$ and the interval length (10), we have

$$
\beta=\frac{1}{E(T)}=\frac{1}{T_{0} E(N)}
$$

It remains to calculate $E(N)=\sum_{n=1}^{\infty} n P[N=n]$. Now

$$
P[N=n]=Q_{n-1}(S-s)-Q_{n}(S-s)
$$

where $Q_{n}$ is the probability distribution function of $S_{n}$ with the convention that $Q_{0}$ is the unit step function. Hence

$$
E(N)=1+H(S-s),
$$

where $H(t)$ is the renewal function

$$
H(t)=\sum_{n=1}^{\infty} \mathbf{Q}_{n}(t) .
$$

From (12) and (14) the average number of stock arrivals per unit time is therefore

$$
\beta=\left\{\mathbf{T}_{0}[1+\mathrm{H}(\mathrm{S}-\mathbf{s})]\right\}^{1}
$$

The density function $f_{X}$ of $X(t)$ is computed separately for the two cases $x \leq s$ and $s<\mathrm{x} \leq \mathrm{S}$. We start with the former. Consider

$$
\begin{aligned}
P[\{x<x(t) & \left.\leq x+d x\} \cap\left\{y<L_{1}(t) \leq y+d y\right\} \cap\{N=n\}\right] \\
& =P\left[\left\{x<s-S_{n} \leq x+d x\right\} \cap\left\{S_{n-1}<S-s \leq S_{n}\right\} \cap A_{0} \cap A_{1}\right]
\end{aligned}
$$

The right-hand side was obtained by inserting the condition for $L_{1}$ in terms of $A_{0}$ and $A_{1}$, and by using (11) to rewrite the set $\{N=n\}$. We pursue the same argument as in (6) and (7) in Example I. More specifically, the probability (17) can be written

$$
\begin{aligned}
\beta \text { dy } P\left[\left\{S-x-d x<S_{n}\right.\right. & \left.\leq S-x\} \cap\left\{S_{n-1} \leq S-s\right\} \cap A_{o} \mid A_{1}\right] \\
& =\beta d y P\left[\left\{S-x-d x<S_{n} \leq S-x\right\} \cap\left\{S_{n} \leq S-s\right\}\right]
\end{aligned}
$$

for $0<y \leq T_{0}$. If $n=1$, the set $\left\{S_{n} \leq S-s\right\}$ does not appear, and so

$$
\begin{aligned}
P[\{x<X(t) & \left.\leq x+d x\} \cap\left\{y<L_{1}(t) \leq y+d y\right\} \cap\{N=1\}\right] \\
& =\beta d y P\left[S-x-d x<Y_{1} \leq S-x\right]=\beta d y q_{1}(S-x) d x .
\end{aligned}
$$


In (19), $q_{n}$ is the probability density for the sum of any $n$ of the $Y_{k}$, that is, $q_{n}(x)=d Q_{n}(x) / d x$. The marginal probability

$$
P[\{x<X(t) \leq x+d x\} \cap\{N=1\}]=\beta T_{0} q_{1}(S-x) d x
$$

is found from (19) by integrating on $y$ from zero to $T_{0}$, where the upper limit is the largest value attainable for $L_{1}(t)$ when $X(t) \leq s$.

Now for $n=2,3, \ldots$, the probability (17) is first developed according to (18). When the right side of (18) is expressed in terms of the probability densities $q_{n}$ there results

$$
\begin{aligned}
P[\{x<X(t) & \left.\leq x+d x\} \cap\left\{y<L_{1}(t) \leq y+d y\right\} \cap\{N=n\}\right] \\
& =\beta d y \int_{u \leq S-S} P\left[\left\{u<S_{n-1} \leq u+d u\right\} \cap\left\{S-x-u<Y_{n} \leq S-x-u+d u\right\}\right] \\
& =\beta d y d x \int_{0}^{S-s} q_{n-1}(u) q_{1}(S-x-u) d u
\end{aligned}
$$

The right side of (21) has used the assumption of mutual independence and identical distributions for the demands $Y_{k}$. The reasoning leading to (20) yields for the marginal probability

$$
P[\{x<X(t) \leq x+d x\} \cap\{N=n\}]=\beta T_{o} d x \int_{0}^{S-s} q_{n-1}(u) q_{1}(S-x-u) d u .
$$

The formula for total probability

$$
P[x<x(t) \leq x+d x]=\sum_{n=1}^{\infty} P[\{x<X(t) \leq x+d x\} \cap\{N=n\}]
$$

can now be applied to the joint probabilities (19) and (22). Thus

$$
f_{X}(x)=\beta T_{0}\left\{q_{1}(s-s)+\int_{0}^{s-s} q_{1}(s-x-u) \sum_{n=1}^{\infty} q_{n}(u) d u\right\}
$$

The sum on the right is the derivative of the renewal function $\mathrm{H}(\mathrm{u})$, and will be denoted by $\mathrm{h}(\mathrm{u})$. The desired result is therefore

$$
f_{X}(x)=\frac{1}{1+H(S-s)}\left\{q_{1}(S-s)+\int_{0}^{S-s} q_{1}(S-s-u) h(u) d u\right\}
$$

by a substitution for $\beta$ from (16).

The second case, $s<x \leq S$, remains to be considered. For this case $n \geq 2$, and $k T_{0}<y \leq(k+1) T_{0}$ for some $k=1,2, \ldots, n-1$. Now 
(26)

$$
\begin{aligned}
P[\{x<X(t) & \left.\leq x+d x\} \cap\left\{y<L_{1}(t) \leq y+d y\right\} \cap\{N=n\}\right] \\
& =P\left[\left\{x<S-S_{n-k} \leq x+d x\right\} \cap\left\{S_{n-1}<S-s \leq S_{n}\right\} \cap A_{0} \cap A_{1}\right] \\
& =\beta \text { dy } P\left[\left\{S-x-d x<S_{n-k} \leq S-x\right\} \cap\left\{S_{n-1}<S-s \leq S_{n}\right\} \cap\{T>y\}\right] .
\end{aligned}
$$

Since $\{\mathbf{T}>\mathrm{y}\}$ is already implied by the intersection of the other sets on the right of (26) by the choice of $k$

$$
\begin{aligned}
P[\{x<x(t) & \left.\leq x+d x\} \cap\left\{y<L_{1}(t) \leq y+d y\right\} \cap\{N=n\}\right] \\
& =\beta d y q_{n-k}(S-x) d x\left[Q_{k-1}(x-s)-Q_{k}(x-s)\right]
\end{aligned}
$$

When the left side of (27) is summed over $n$ (starting with $n=k+1$ because $k+1$ is the minimum value that can be assumed by $N$ ) the total probability law yields

$$
\begin{aligned}
P[\{x<X(t) & \left.\leq x+d x\} \cap\left\{y<L_{1}(t) \leq y+d y\right\}\right] \\
& =\beta d y d x\left[Q_{k-1}(x-s)-Q_{k}(x-s)\right] h(S-x) .
\end{aligned}
$$

As before, $h(u)=\sum_{1}^{\infty} q_{n}(u)$. Next, let us integrate (28) on y over the interval $\left(k T_{0},\{k+1\} T_{0}\right]$; the integration leads to

$$
\begin{aligned}
P[\{x<x(t) & \left.\leq x+d x\} \cap\left\{k T_{0}<L_{1}(t) \leq(k+1) T_{0}\right\}\right] \\
& =\beta T_{0}\left[Q_{k-1}(x-s)-Q_{k}(x-s)\right] h(S-x) .
\end{aligned}
$$

Finally, the marginal density of $X(t)$ is found from (29) via the total probability law by summing on $k$. Since

$$
\sum_{k=1}^{\infty}\left[Q_{k-1}(x-s)-Q_{k}(x-s)\right]=1
$$

we obtain $f_{X}(x)=\beta T_{0} h(S-x)$. The final result is therefore

$$
f_{X}(x)= \begin{cases}{[1+H(S-s)]^{-1} h(s-x)} & \text { for } s<x \leq S, \\ {[1+H(S-s)]^{-1}\left\{q_{1}(S-x)+\int_{0}^{S-s} q_{1}(S-x-u) h(u) d u\right\}} & \text { for } x \leq s,\end{cases}
$$

where $f_{X}(x)$ for $x \leq s$ has been previously obtained in (25). 


\section{EXAMPLE III: A CONTINUOUS TIME TWO-BIN SYSTEM WITH}

\section{BACKLOG AND LINEAR DEPLETION}

This example is a more sophisticated version of Example $I$; introduction of a time lag in delivery and service from a second bin in the interim are complicating features of the present example.

Imagine that the stock on hand is stored in two bins labeled respectively A and B. Demand is met by service from bin $A$ until the stock in bin $A$ is exhausted. An order for stock replenishment is then placed, but delivery occurs only after a random time lag $\tau$. During this delay the demand is met by service from bin $B$, with negative stock supplies being regarded as owed to demand. When delivery is made, bin $A$ contains a random quantity of stock, $M$, while bin $B$ has a fixed (nonrandom) quantity $s$. Depletion from stock is at a random linear rate $\lambda$ per unit time. It is assumed that $M, \tau$, and $\lambda$ are mutually independent within each reordering period, but not necessarily from period to period.

What is the probability density of $X(t)$, the total stock on hand at time $t$ ?

\section{SOLUTION}

In its equilibrium state, the stock on hand is a stationary random process as depicted in Figure 3. The random times $t_{n}$ of stock arrival constitute an s.p.p. and the time interval $T$ separating two consecutive intervals is a random variable

$$
\mathrm{T}=\mathrm{M} \lambda^{-1}+\tau
$$

Here $T$ may be taken as any such interval, $M$ the stock in bin $A$ on delivery, and $\lambda$ the depletion rate for this interval; this follows because the statistics of these variables are the same in each interval. We shall again require the average number of stock arrivals per unit time $\beta$. From (3) and (31) immediately preceding,

$$
\beta=\frac{1}{E(T)}=\left[E(M) E\left(\lambda^{-1}\right)+E(\tau)\right]^{-1} .
$$

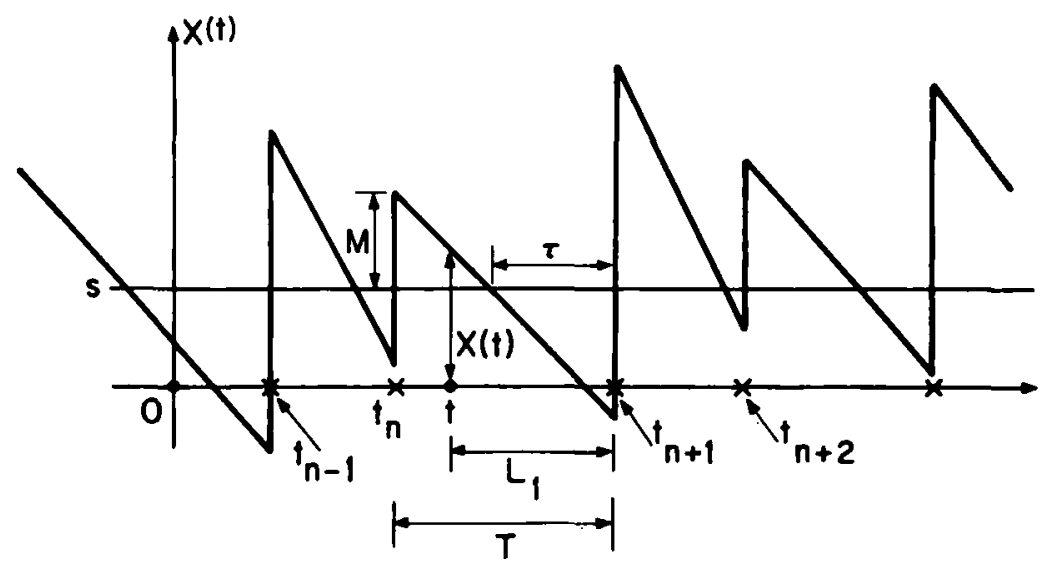

Figure 3. Two-bin inventory system with backlog and linear random depletion 
The remainder of the procedure for finding $f_{X}$ follows the now familiar lines of the earlier examples. Again, $x \leq s$ and $x>s$ are considered separately. When $x \leq s$, we have for $\mathrm{y} \leq \mathrm{z}$

$$
\begin{aligned}
\mathbf{P}[\{x<X(t) & \left.\leq x+d x\} \cap\left\{y<L_{1}(t) \leq y+d y\right\} \cap\{z<\tau \leq z+d z\}\right] \\
& =P\left[\{x<s-\lambda(z-y) \leq x+d x\} \cap\{z<\tau \leq z+d z\} \cap A_{0} \cap A_{1}\right] \\
& =P\left(A_{1}\right) P[\{x<s-\lambda(z-y) \leq x+d x\} \cap\{T>y\} \cap\{z<\tau \leq z+d z\}] \\
& =\beta \text { dy } P[\{x<s-\lambda(z-y) \leq x+d x\} \cap\{z<\tau \leq z+d z\}] \\
& =\beta \text { dy } f_{\lambda}\left(\frac{s-x}{z-y}\right) \frac{d x}{z-y} f_{\tau}(z) d z,
\end{aligned}
$$

where $f_{\lambda}$ and $f_{\tau}$ are the probability density functions of $\lambda$ and $\tau$, respectively. For $y>z$ the probability (33) is zero. The remainder of the computation is also like that of the earlier examples. We determine $f_{X}$ as a marginal probability by integrating on $y$ from zero to $z$ and then on $\mathrm{z}$ from zero to infinity, viz.

$$
\begin{aligned}
f_{X}(x) & =\beta \int_{0}^{\infty} f_{\tau}(z) d z \int_{0}^{z} \frac{1}{z-y} f_{\lambda}\left(\frac{s-x}{z-y}\right) d y \\
& =\beta \int_{0}^{\infty} f_{\tau}(z) d z \int_{0}^{z} u^{-1} f_{\lambda}\left(\frac{s-x}{u}\right) d u
\end{aligned}
$$

The case $x>s$ is treated similarly. The probability in question is zero for $y \leq z$, and for $y>z$

(35) $P\left[\{x<X(t) \leq x+d x\} \cap\left\{y<L_{1}(t) \leq y+d y\right\} \cap\{z<\tau \leq z+d z\}\right]$

$$
\begin{aligned}
& =\beta \text { dy } P[\{x<s+\lambda(y-z) \leq x+d x\} \cap\{T>y\} \cap\{z<\tau \leq z+d z\}] \\
& =\beta \text { dy } \mathbf{P}\left[\left\{\frac{\mathbf{x}-\mathbf{s}}{\mathbf{y}-\mathbf{z}}<\lambda \leq \frac{\mathbf{x}-\mathbf{s}}{\mathbf{y}-\mathbf{z}}+\frac{\mathrm{dx}}{\mathbf{y}-\mathbf{z}}\right\} \cap\left\{\frac{\mathbf{M}}{\lambda}+\tau>\mathbf{y}\right\} \cap\{\mathbf{z}<\tau \leq \mathbf{z}+\mathrm{dz}\}\right] \\
& =\beta \text { dy } P\left[\left\{\frac{\mathbf{x}-\mathbf{s}}{\mathbf{y}-\mathbf{z}}<\lambda \leq \frac{\mathbf{x}-\mathbf{s}}{\mathbf{y}-\mathbf{z}}+\frac{\mathbf{d x}}{\mathbf{y}-\mathbf{z}}\right\} \cap\{\mathbf{M}>\mathbf{x}-\mathbf{s}\} \cap\{\mathbf{z}<\tau \leq \mathbf{z}+\mathbf{d z}\}\right] \\
& =\beta d y f_{\lambda}\left(\frac{x-s}{y-z}\right) \frac{d x}{y-z}\left[1-F_{M}(x-s)\right] f_{\tau}(z) d z
\end{aligned}
$$

in which $F_{M}$ stands for the distribution function of $M$. Thus the probability density for $X(t)$ is 


$$
\begin{aligned}
f_{X}(x) & =\beta\left[1-F_{M}(x-s)\right] \int_{0}^{\infty} f_{\tau}(z) d z \int_{z}^{\infty} \frac{1}{y-z} f_{\lambda}\left(\frac{x-s}{y-z}\right) d y \\
& =\beta\left[1-F_{M}(x-s)\right] \int_{0}^{\infty} f_{\tau}(z) d z \int_{0}^{\infty} u^{-1} f_{\lambda}(u) d u \\
& =\beta E\left(\lambda^{-1}\right)\left[1-F_{M}(x-s)\right]
\end{aligned}
$$

for $x>s$. The latter may be combined with $f_{x}$ for $x \leq s$ from (34) and the expression (32) for $\beta$ to yield

$$
f_{X}(x)= \begin{cases}{\left[E(M) E\left(\lambda^{-1}\right)+E(\tau)\right]^{-1} \int_{0}^{\infty} f_{\tau}(z) d z \int_{0}^{\infty} u^{-1} f_{\lambda}\left(\frac{s-x}{u}\right) d u} & \text { for } x \leq s \\ {\left[E(M) E\left(\lambda^{-1}\right)+E(\tau)\right]^{-1} E\left(\lambda^{-1}\right)\left[1-F_{M}(x-s)\right]} & \text { for } x>s\end{cases}
$$

In particular, if the depletion rate $\lambda$ is a constant, $f_{\lambda}(v)=\delta(\lambda-v)$ (i.e., a delta function) and consequently

$$
\mathrm{f}_{X}(\mathrm{x})= \begin{cases}{[E(M)+\lambda E(\tau)]^{-1}\left[1-F_{\tau}\left(\frac{s-x}{\lambda}\right)\right]} & \text { for } x \leq s \\ {[E(M)+\lambda E(\tau)]^{-1}\left[1-F_{M}(x-s)\right]} & \text { for } x>s\end{cases}
$$

\section{EXAMPLE IV: A CONTINUOUS TIME TWO-BIN SYSTEM WTTH RANDOM PARAMETER POISSON DEPLETION}

The subject inventory system is a two-bin model resembling that of Example III. In place of linear depletion, the demand on the stock on hand $X(t)$ takes the form of unit decrements which constitute a simple Poisson process; during each reordering cycle, the Poisson parameter $\lambda$ is a random variable having the (same) probability density $f_{\lambda}$. Since we suppose that $s$ is an integer, and $M$ is an integer-valued random variable, the stock on hand $X(t)$ is likewise integer-valued, as shown in Figure 4. We then ask: what is $P[X(t)=k]$ ?

\section{SOLUTION}

If $T$ is any period between successive deliveries, $T_{i}$ the time interval between the (i-1)st and $i^{\prime}$ th unit demand, and $\tau$ is the (random) lag between ordering and delivery

$$
\mathbf{T}=\mathbf{T}_{1}+\mathbf{T}_{2}+\cdots+\mathbf{T}_{\mathbf{M}}+\tau
$$

Because the depletion is a simple Poisson process the $T_{i}$ are mutually independent, and $f_{T_{i}}(w)=\lambda e^{-\lambda w}$ for each. Hence 


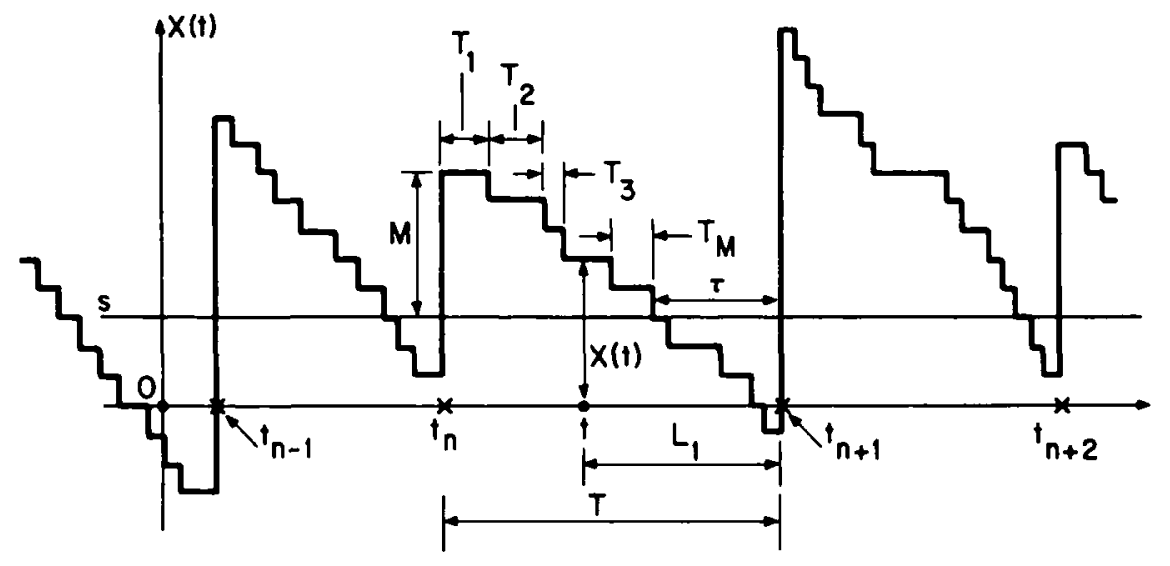

Figure 4. Continuous time two-bin inventory system with random parameter Poisson depletion

$$
\mathrm{f}_{\mathrm{T}_{\mathrm{i}}}(\mathrm{w})=\int_{0}^{\infty} \nu \mathrm{e}^{-\lambda \nu} \mathrm{f}_{\lambda}(\nu) \mathrm{d} \nu
$$

and

$$
E\left(T_{i}\right)=E\left(\lambda^{-1}\right)
$$

It then follows from (41) and (39) that the average rate of stock arrivals is

$$
\beta=\frac{1}{E(T)}=\left[E(M) E\left(\lambda^{-1}\right)+E(\tau)\right]^{-1},
$$

just as in Example III [cf. (32)].

To calculate $P[X(t)=s+k]$ it is necessary to distinguish between the two cases $k$ negative and positive. For the former, consider $P[X(t)=s-m], m=0,1,2, \ldots$, with $y \leq z$ :

$$
\begin{aligned}
& \mathbf{P}\left[\{\mathrm{X}(\mathrm{t})=\mathbf{s}-\mathbf{m}\} \cap\left\{\mathbf{y}<\mathbf{L}_{1}(\mathrm{t}) \leq \mathrm{y}+\mathrm{dy}\right\} \cap\{\mathrm{z}<\tau \leq \mathrm{z}+\mathbf{d z}\} \cap\{\mathbf{w}<\lambda \leq \mathrm{w}+\mathbf{d w}\}\right] \\
& =\beta \text { dy } P\left[E_{m} \cap\{\mathbf{T}>y\} \cap\{z<\tau \leq z+d z\} \cap\{w<\lambda \leq w+d w\}\right] \\
& =\beta \text { dy } P\left[E_{m} \cap\{z<\tau \leq z+d z\} \cap\{w<\lambda \leq w+d w\}\right] \\
& =\beta d y \frac{[w(z-y)]^{m} \exp [-w(z-y)]}{m !} f_{\tau}(z) d z f_{\lambda}(w) d w .
\end{aligned}
$$

In the above, $E_{m}$ denotes the event " $m$ demands in the interval $(t, t+z-y]$," and the probability in the last step is a consequence of the independence of $\tau$ from the $T_{i}$, together with $\mathbf{P}\left[\mathbf{E}_{\mathrm{m}} \cap\{w<\lambda \leq w+d w\}\right]=\mathbf{P}\left[\mathbf{E}_{\mathrm{m}} \mid \lambda=w\right] \mathrm{f}_{\lambda}(w)$ dw. The first two steps in the computation (43) follow similar reasoning used in the preceding examples. For $y>z$ the probability on the left of (43) is zero, and so the marginal probability $P[X(t)=s-m]$ is obtained from (43) by integrating the right side on $y$ from zero to $z$, and on $z$ and $w$ from zero to infinity. With the change of variable $u=z-y$, the result of these computations is 


$$
\begin{aligned}
P[X(t)=s-m] & =\beta \int_{0}^{\infty} f_{\lambda}(w) d w \int_{0}^{\infty} f_{\tau}(z) d z \int_{0}^{z} \frac{[w u]^{m}}{m !} e^{-w u} d u \\
& =\beta \int_{0}^{\infty} w^{-1} f_{\lambda}(w) d w \int_{0}^{\infty} f_{\tau}(z)\left\{1-\left[\sum_{n=0}^{m} \frac{(w z)^{n}}{n !} e^{-w z}\right]\right\} d z .
\end{aligned}
$$

When $X(t)=s+m, m=1,2, \ldots$, the stock at the beginning of the reordering period must be at least $m$, i.e., $M=m+n$ with $n$ a non-negative integer. Thus for each $n=0,1, \ldots$, we consider separately

(45)

$$
\begin{aligned}
& \mathbf{P}\left[\{\mathbf{X}(\mathrm{t})=\mathbf{s}+\mathbf{m}\} \cap\left\{\mathbf{y}<\mathrm{L}_{1}(\mathrm{t}) \leq \mathrm{y}+\mathrm{dy}\right\} \cap\{\mathbf{z}<\tau \leq \mathbf{z}+\mathrm{dz}\} \cap\{\mathbf{w}<\lambda \leq \mathrm{w}+\mathrm{dw}\} \cap\{\mathbf{M}=\mathbf{m}+\mathbf{n}\}\right] \\
& =\beta \text { dy } P\left[E_{m} \cap\{z<\tau \leq z+d z\} \cap\{w<\lambda \leq w+d w\} \cap\{M=m+n\}\right] \\
& =\beta \text { dy } P\left[\{ T _ { n + 1 } + \cdots + T _ { m + n - 1 } < y - z \leq T _ { n + 1 } + \cdots T _ { m + n } \} \cap \left\{T_{1}+\cdots+T_{m+n}\right.\right. \\
& +\tau>\mathrm{y}\} \cap\{\mathrm{z}<\tau \leq \mathrm{z}+\mathrm{dz}\} \cap\{\mathrm{w}<\lambda \leq \mathrm{w}+\mathrm{dw}\} \cap\{\mathbf{M}=\mathbf{m}+\mathrm{n}\}\} \\
& =\beta \text { dy } P\left[\left\{T_{n+1}+\cdots+T_{m+n-1}<y-z \leq T_{n+1}+\cdots+T_{m+n}\right\} \cap\{z<\tau \leq z+d z\} \cap\right. \\
& n\{w<\lambda \leq w+d w\} \cap\{\mathbf{M}=\mathbf{m}+\mathbf{n}\}] \\
& =\beta d y \frac{[w(y-z)]^{m} \exp [-w(y-z)]}{m !} f_{\tau}(z) d z f_{\lambda}(w) d w P[M=m+n] .
\end{aligned}
$$

The above holds only for $y>z$; for $y \leq z$ the probability (45) is zero. We once more integrate on the variables $y, z$, and $w$ to find the marginal probability. The integration is facilitated if we take $u=y-z$, thus obtaining an integral on $u$ instead of $y$ with new limits from zero to infinity (rather than $z$ to infinity). The result is then

$$
\begin{aligned}
P[\{X(t)=s+m\} \cap\{M & =m+n\}] \\
& =\left\{\beta \int_{0}^{\infty} f_{\lambda}(w) d w \int_{0}^{\infty} f_{\tau}(z) d z \int_{0}^{\infty} \frac{[w u]^{m}}{m !} e^{-w u} d u\right\} P[M=m+n] .
\end{aligned}
$$

The integration on $u$ is easily performed. Further, the total probability law can be applied to (46) by summing both sides for $n=0,1,2, \ldots$. On the right side this summation yields

$$
\sum_{n=0}^{\infty} P[M=m+n]=P[M \geq m]=1-P[M \leq m-1]=1-F_{M}(m-1)
$$


where $F_{M}$ is the probability distribution function for $M$. When the indicated steps are carried out, we have

(48)

$$
\begin{aligned}
& \mathrm{P}[\mathrm{X}(\mathrm{t})=\mathrm{s}+\mathrm{m}]=\left\{\beta \int_{0}^{\infty} \mathrm{f}_{\lambda}(\mathrm{w}) \mathrm{dw} \int_{0}^{\infty} \mathrm{f}_{\tau}(\mathrm{z}) \mathrm{dz} \mathrm{w}^{-1}\right\}\left[1-\mathrm{F}_{M}(\mathrm{~m}-1)\right] \\
& =\left\{\beta \int_{0}^{\infty} w^{-1} f_{\lambda}(w) d w\right\}\left[1-F_{M}(m-1)\right]=\beta E\left(\lambda^{-1}\right)\left[1-F_{M}(m-1)\right] .
\end{aligned}
$$

Our calculations may then be summarized by combining the expression (48) for positive integers with (44) for negative integers, viz.,

$$
\begin{aligned}
& {\left[E(M) E\left(\lambda^{-1}\right)+E(\tau)\right]^{-1} \int_{0}^{\infty} f_{\lambda}(w) d w} \\
& P[X(t)=s+k]=\left\{\begin{array}{rr}
\quad x \int_{0}^{\infty} f_{\tau}(z)\left\{1-\left[\sum_{n=0}^{-k} \frac{(w z)^{-n}}{(-n) !} e^{-w z}\right]\right\} d z & \text { for } k=0,-1,-2, \ldots \\
{\left[E(M) E\left(\lambda^{-1}\right)+E(\tau)\right]^{-1} E\left(\lambda^{-1}\right)\left[1-F_{M}(k-1)\right]} & \text { for } k=1,2, \ldots .
\end{array}\right.
\end{aligned}
$$

A constant (nonrandom) depletion rate $\lambda$ simplifies (49) to

(50)

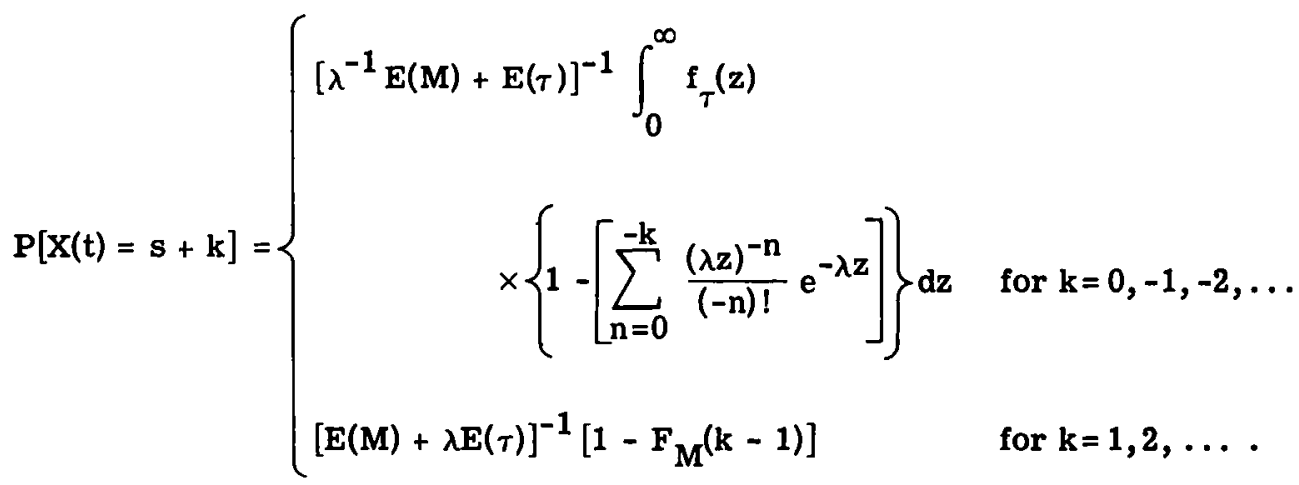

\section{REFERENCES}

[1] Arrow, K. J., Karlin, S., and Scarf, H., Studies in the Mathematical Theory of Inventory and Production (Stanford Univ. Press, Stanford, Calif., 1958).

[2] Beutler,-F. J. and Leneman, O. As Z., "The Theory of Stationary Point Processes," in Acta Math., $-116,159-197 .(1966)$. 
[3] Beutler, F. J. and Leneman, O. A. Z., "Random Sampling of Random Processes," Information and Control, $\underline{9}, 325-346$ (1966).

[4] Bulinskaya, E., "Steady-State Solutions in Problems of Optimum Inventory Control," in Theory of Prob. and Its Appl., 9, 502-507 (1964).

[5] Scarf, H., Gilford, D., and Shelly, M., Multistage Inventory Models and Techniques (Stanford Univ. Press, Stanford, Calif., 1963). 